\title{
Electronic and Magnetic Properties of the Interface $\mathrm{LaAlO}_{3} / \mathrm{TiO}_{2}$ Anatase from Density Functional Theory
}

\author{
Mariana Weissmann and V. Ferrari \\ Gerencia de Investigación y Aplicaciones, CNEA, Avenida General Paz 1499, San Martín, 1650 Buenos Aires, Argentina
}

Correspondence should be addressed to Mariana Weissmann, weissman@cnea.gov.ar

Received 16 February 2012; Accepted 7 April 2012

Academic Editor: Carlos Martinez-Boubeta

Copyright ( 92012 M. Weissmann and V. Ferrari. This is an open access article distributed under the Creative Commons Attribution License, which permits unrestricted use, distribution, and reproduction in any medium, provided the original work is properly cited.

\begin{abstract}
$\mathrm{Ab}$ initio calculations using the local spin density approximation and also including the Hubbard $\mathrm{U}$ have been performed for three low-energy configurations of the interface between $\mathrm{LaAlO}_{3}$ and $\mathrm{TiO}_{2}$ anatase. Two types of interfaces have been considered: $\mathrm{LaO} / \mathrm{TiO}_{2}$ and $\mathrm{AlO}_{2} / \mathrm{TiO}$, the latter with Ti termination and therefore a missing oxygen. A slab-geometry calculation was carried out, and all the atoms were allowed to relax in the direction normal to the interface. In all the cases considered, the interfacial $\mathrm{Ti}$ atom acquires a local magnetic moment, and its formal valence is less than +4 . When there are oxygen vacancies, this valence decreases abruptly inside the anatase slab, while in the $\mathrm{LaO} / \mathrm{TiO}_{2}$ interface, the changes are more gradual.
\end{abstract}

\section{Introduction}

Complex oxide heterostructures have been the subject of many recent papers, both experimental and theoretical, as their interesting interface properties promise to pave the way towards novel electronic devices. With the current available experimental techniques, oxide thin films can be produced with a high degree of crystallinity, and the electronic structure of their surfaces and interfaces can be precisely determined. The most studied system of this type has been $\mathrm{LaAlO}_{3} / \mathrm{SrTiO}_{3}(\mathrm{LAO} / \mathrm{STO})$ that consists of two perovskite structures stacked along the (001) direction [1, 2 ], in which they present alternate layers of $\mathrm{LaO}, \mathrm{AlO}_{2}$, $\mathrm{TiO}_{2}$, and $\mathrm{SrO}$ with a very small lattice mismatch between them. A similar but less studied interface is that of LAO with $\mathrm{TiO}_{2}$ anatase that presents an even smaller lattice mismatch, namely less than $0.1 \%$, along the (001) direction [3]. $\mathrm{TiO}_{2}$ is a key material for most applications, including catalytic and optical devices, sensors, optoelectronics, and spintronics. Anatase thin films are frequently grown over LAO by pulsed laser deposition. It seems therefore important to perform a careful characterization, both from theory and experiments, of the film/substrate interface. Just to mention one example where this study may be relevant, room temperature ferromagnetism has been obtained from doped and undoped anatase films grown over LAO, and the results strongly depend on the growth conditions [4].

There are two possible interfaces for the system LAO/ $\mathrm{TiO}_{2}$-anatase, namely, $\mathrm{LaO}$ facing $\mathrm{TiO}_{2}$ and $\mathrm{AlO}_{2}$ facing $\mathrm{TiO}_{2}$ $[5,6]$. Due to the ionicity of the component oxides and, in particular, to the fact that the layers of $\mathrm{LAO}\left(\mathrm{AlO}_{2}\right.$ and $\left.\mathrm{LaO}\right)$ have alternating formal charges $(-1$ and +1 , resp.), there is an interfacial formal excess charge that should be compensated either by the presence of terraces with different stacking, or by oxygen vacancies or by atomic interdiffusion.

Experimental work [6] has shown that, in the case of $\mathrm{LAO} / \mathrm{TiO}_{2}$-anatase, terraces with both types of interfaces appear and approximately in the same proportion. For the theoretical modeling of this system, the fact that the $\mathrm{TiO}_{2}$ anatase layers along the (001) direction are not strictly planar must be taken into account. As a consequence, the anatase interface can be either oxygen terminated and thus neutral (with formal valence +4 for the interfacial $\mathrm{Ti}$ ion), or Titerminated. The latter situation is equivalent to considering surface oxygen vacancies and gives rise to a change in the valence of the interfacial Ti ion. 


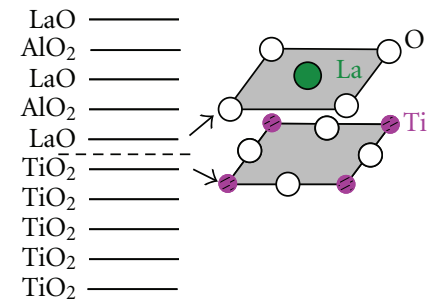

(a) $\mathrm{LaO} / \mathrm{TiO}_{2}$

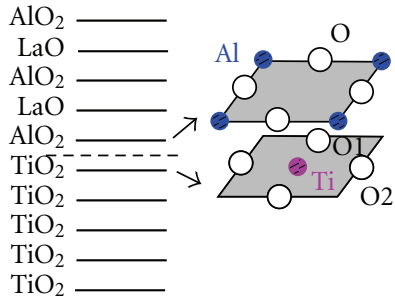

(b) $\mathrm{AlO}_{2}$ hollow

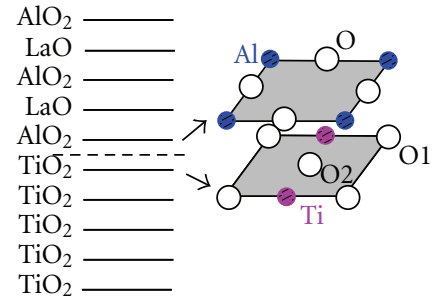

(c) $\mathrm{AlO}_{2}$ bridge

Figure 1: Slab structures for three low energy configurations. (a) interface $\mathrm{LaO} / \mathrm{TiO}_{2}$; (b) interface $\mathrm{AlO} / 2$ TiO, with $\mathrm{Ti}$ atom in the hollow position; (c) interface $\mathrm{AlO}_{2} / \mathrm{TiO}$, with Ti atom in the bridge position. Oxygen atoms are coplanar with La and $\mathrm{Al}$ ions and $0.4 \AA$ off-plane in the Ti layers. $\mathrm{O} 1$ and $\mathrm{O} 2$ are below and above the Ti plane, respectively. Oxygen $\mathrm{O} 2$ is closer to LAO, and it is removed in cases (b) and (c).

In this paper, we study the electronic and magnetic properties of interfaces for different stackings of $\mathrm{LAO} / \mathrm{TiO}_{2}$ using density functional theory [7] in the local density approximation (LSDA) and also considering electronic correlations in the framework of the $\mathrm{LSDA}+\mathrm{U}$ approximation.

\section{Method of Calculation}

To simulate the interfaces, we use a periodically repeated slab geometry with 5 layers of each of the component materials and enough empty space between slabs so that they do not interact with each other (see Figure 1). The cell parameters in the plane of the interface are kept fixed to those of the experimental bulk LAO (3.79 $\AA$ ) [3], and the positions of all atoms are allowed to relax in the out-of-plane direction until the forces are smaller than $0.05 \mathrm{eV} / \AA$.

As in our previous work $[5,8]$, we use an odd number of layers of LAO to avoid the formation of a large dipole moment, but we are aware that with this procedure it is difficult to assure whether the interface will be conducting or not. The reason lies in the fact that an LSDA calculation for a positively (negatively) charged system will locate the Fermi level in the conduction (valence) band.

We use the Wien $2 \mathrm{k}$ code, that is, an implementation of the full-potential linear-augmented plane waves method (FP-LAPW) [9]. The calculations are scalar relativistic, and the parameters used are listed in [10]. It is well known that in the case of oxides there is a band gap underestimation when using local exchange correlation functionals within density functional methods. For this reason, we have performed calculations with both the LSDA and the LSDA+U approximations [11], using $U=0.4$ Ry for the Ti $d$ orbitals $[8,12]$, as in previous works. The comparison between the two procedures will evidence whether there is a qualitative difference in the interfacial properties due to electron correlations as, for example, a change in the valence of the Ti ions.

\section{Results}

The total energies of different possible structures (at $0 \mathrm{~K}$ and with collinear spins) have been evaluated in previous works $[5,6]$, and in this paper, we present the results for the lowest energy ones. For the interface in which $\mathrm{LaO}$ faces $\mathrm{TiO}_{2}$ (Figure 1(a)), the oxygen termination for anatase is preferred. However, when $\mathrm{AlO}_{2}$ faces $\mathrm{TiO}_{2}$, the lower energy is for the Ti termination, thus with oxygen vacancies $[5,6]$. Two types of Ti-terminated interfaces are studied: one where the interfacial $\mathrm{Ti}$ atom faces the hollow site of the $\mathrm{Al}$ atoms in the $\mathrm{AlO}_{2}$ layer (Figure 1(b)) and another one where the Ti atom faces a bridge position of the $\mathrm{Al}$ sites in the $\mathrm{AlO}_{2}$ plane (Figure 1(c)).

In all the cases considered, there is a total formal charge of +1 arising in (a) from the LAO slab, while in cases (b) and (c), it is due to the contribution of both the LAO slab and the oxygen vacancy in the anatase slab. We point out that there are more possible structures for each type of stacking, and that in this work, we consider three of the lowest-energy ones, those that require more energy to separate $\mathrm{LAO}$ from anatase. $\mathrm{LaO} / \mathrm{TiO}_{2}$ has the lowest energy, followed by $\mathrm{AlO}_{2} / \mathrm{TiO}$ where oxygen vacancies are present [13] (i.e., $\mathrm{O} 2$ is removed in Figures $1(\mathrm{~b})$ and $1(\mathrm{c})$ ). In the hollow structure, one oxygen atom from the $\mathrm{AlO}_{2}$ layer moves towards the interfacial $\mathrm{Ti}$, thus increasing its number of neighbors. This relaxation decreases the total energy of the system with respect to the bridge structure by $0.2 \mathrm{eV}$ in LSDA and $0.4 \mathrm{eV}$ in LSDA+U.

Figure 2 shows the densities of states (DOSs) for the three structures depicted in Figure 1. We present the results for the LSDA+U approximation as they are similar to the LSDA ones, and if there are any differences, they are mentioned in the text. On the left side of Figure 2, we present the total DOS and the partial contributions from the La atoms. On the right side, the DOSs projected on the Ti atoms are shown in a zoomed-in region close to the Fermi energy $\left(E_{f}\right)$. As in the case of pure anatase, the valence band is mostly from the oxygen atoms and the conduction band from the $\mathrm{Ti}$ atoms, with the $d$ orbitals clearly separated by symmetry: the $t_{2} g$ levels closer to $E_{f}$ and the $e_{g}$ ones at higher energies. There are no $\mathrm{Al}$ states close to $E_{f}$, but oxygen atoms from LAO contribute significantly to the higher-energy part of the valence band.

3.1. $\mathrm{LaO} / \mathrm{TiO}_{2}$ Interface. In this interface (Figure 1(a)), $E_{f}$ is in the conduction band, as expected due to the formal charge in the LAO slab. The largest contribution to the occupied states close to $E_{f}$ comes from the interfacial $\mathrm{Ti}$ ion, and the rest of the Ti atoms contribute less as the 

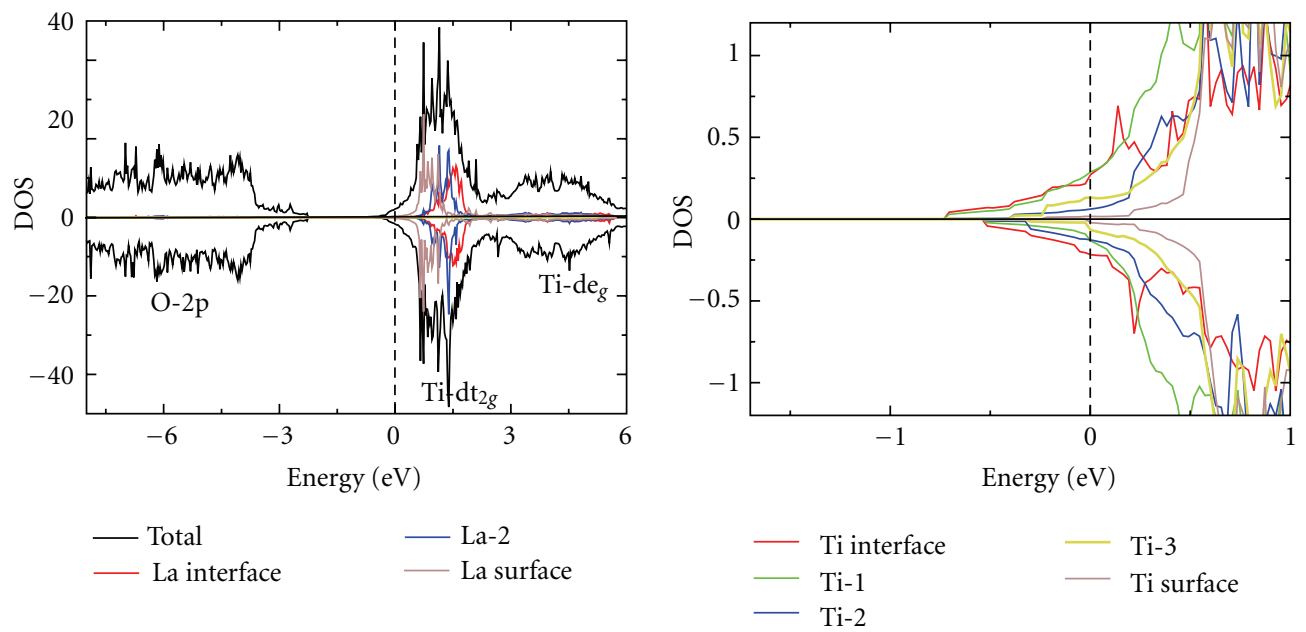

(a) $\mathrm{LaO} / \mathrm{TiO}_{2}$
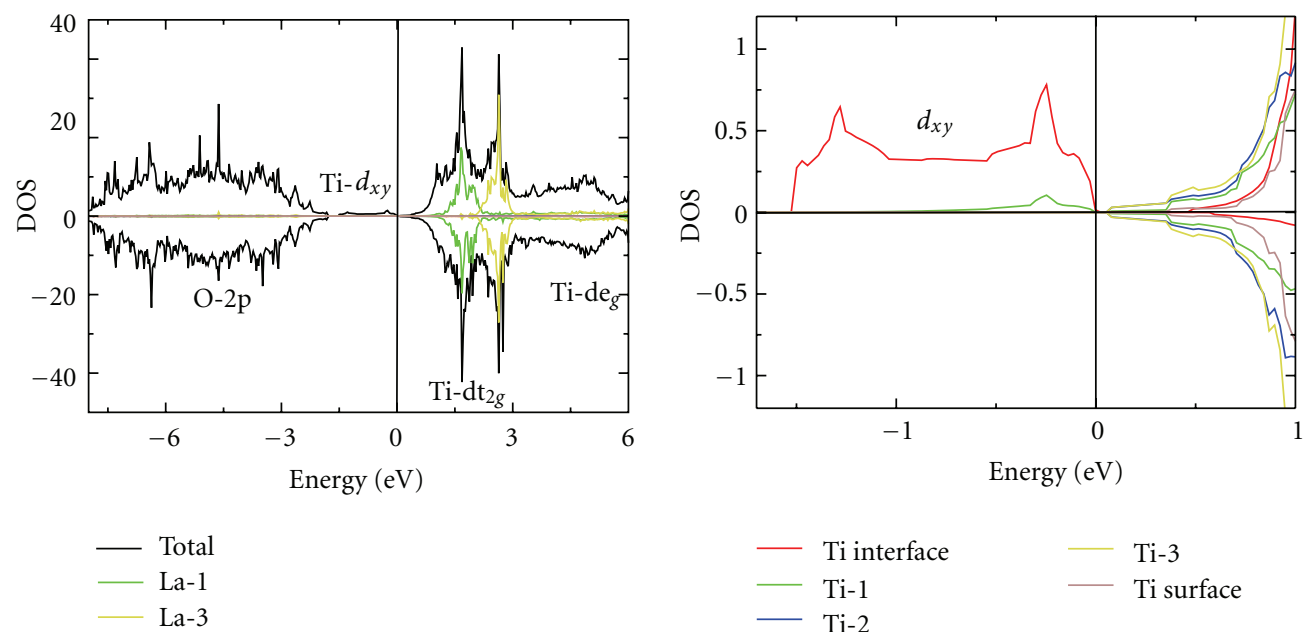

(b) $\mathrm{AlO}_{2}$ hollow
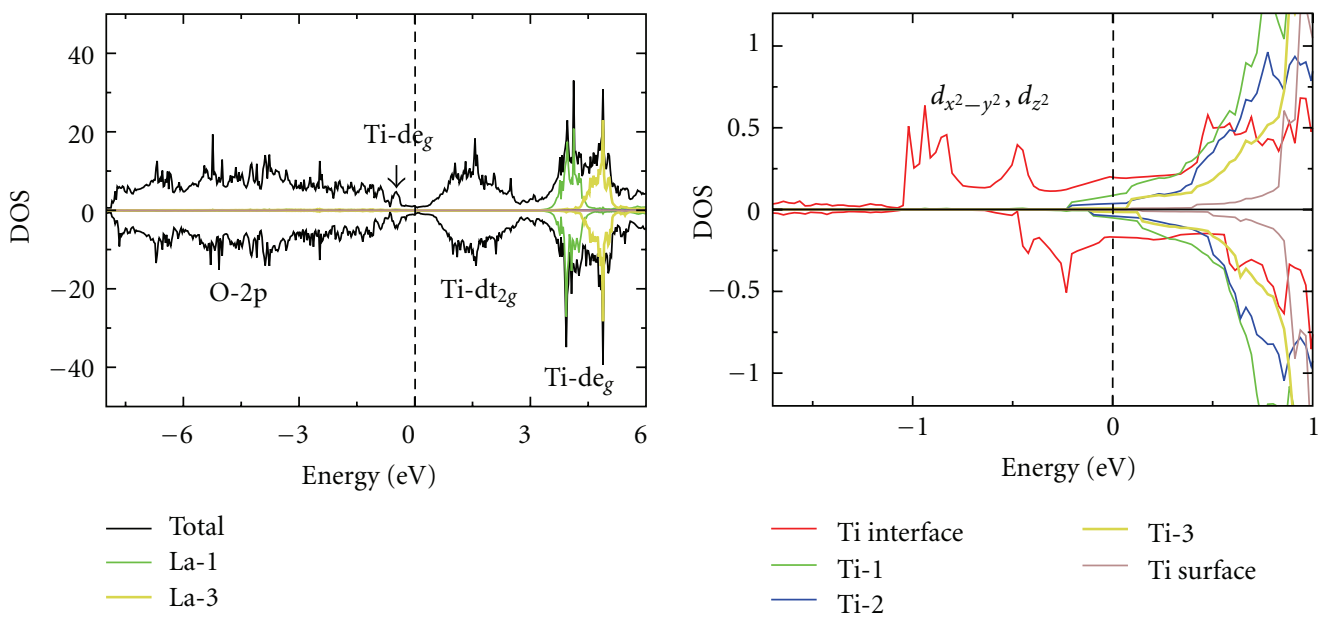

(c) $\mathrm{AlO}_{2}$ bridge

FIgURE 2: Densities of states for the three slabs of Figure 1 in the LSDA+U approximation. (a) Top: $\mathrm{LaO}_{/} \mathrm{TiO}_{2}$, (b) middle: $\mathrm{AlO}_{2}$ hollow, (c) bottom: $\mathrm{AlO}_{2}$ bridge. The Fermi level $\left(E_{f}\right)$ is marked by the dotted vertical line. Positive and negative values correspond to majority and minority spins, respectively. Left: total densities of states of the slabs and La levels. Right: partial DOS on the Ti atoms zoomed-in around $E_{f}$. In the legend the numbers by the atoms indicate the number of planes away from the interface. 


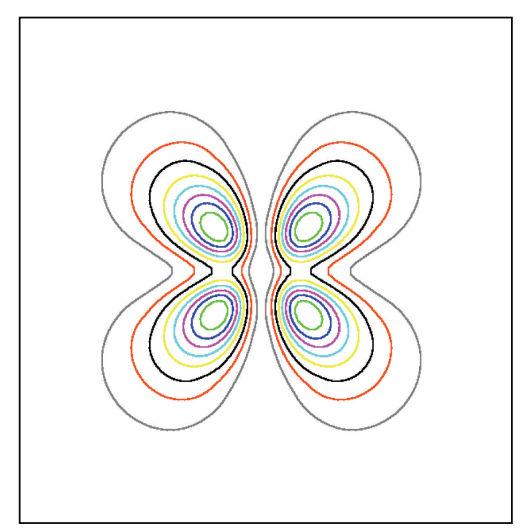

(a)

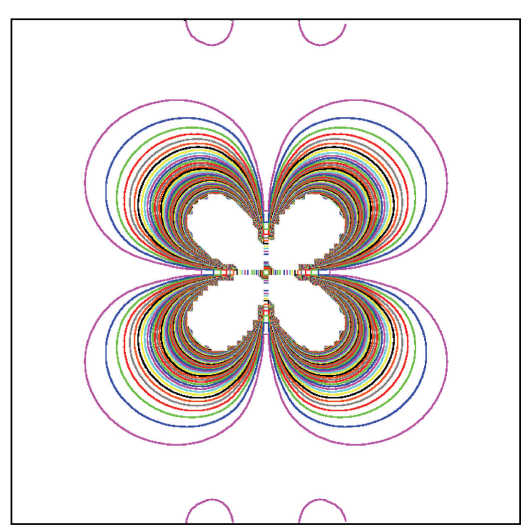

(b)

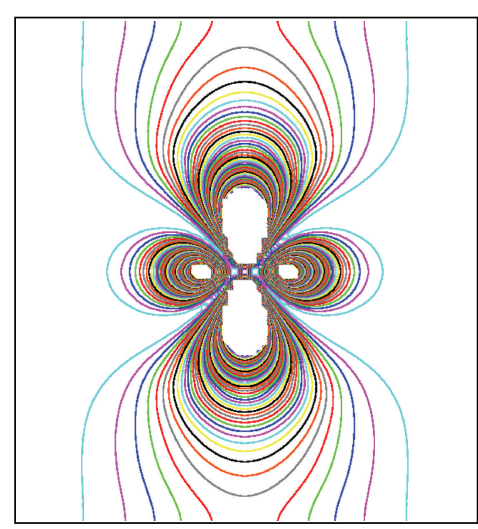

(c)

Figure 3: LSDA+U charge density of states close to $E_{f}$ in the plane defined by the Ti-Ti bonds for one unit cell. The occupied states in the energy range: $\left[E_{f}-1.5 \mathrm{eV}, E_{f}\right]$ are plotted for the three structures in Figure 1, namely, (a) $\mathrm{LaO} / \mathrm{TiO}_{2}$, (b) $\mathrm{AlO}_{2}$ hollow, and (c) $\mathrm{AlO}_{2}$ bridge. Charge isolines are spaced 0.01 , from 0.01 to 1.00 . In each case, the Ti interfacial atom is placed at the center of the figure, so as to make them comparable.

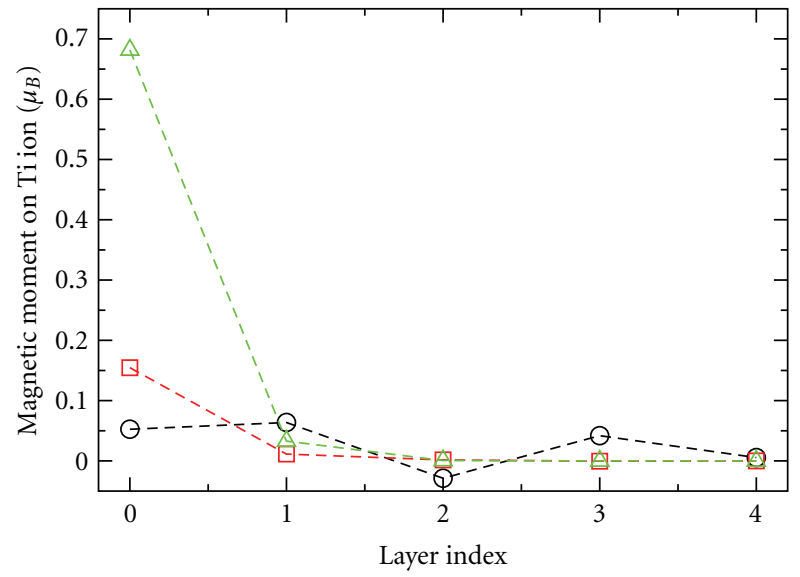

$\ominus \ominus \mathrm{LaO}$

$\square \boxminus$ Bridge

$\Delta \triangle$ Hollow

FIGURE 4: Magnetic moment inside the Ti muffin-tin spheres as a function of the penetration in the anatase slab. The interface is at layer 0 , and the surface is at layer 4 .

distance to the interface increases, as shown in Figure 2 (top right). This is true for both LSDA and LSDA+U approximations. It is interesting to note that similar layerprojected DOSs were obtained for LAO/STO [14] even though in that structure, the $\mathrm{TiO}_{2}$ planes alternate with $\mathrm{SrO}$ layers. The authors also find that the charge distribution is not affected by the inclusion of correlation. There is certainly no contribution from the interfacial La ions to the occupied states, as that peak is shifted towards the right when compared with the La atom at the free surface of the slab (see Figure 2(a)). The charge density at the interface is plotted in Figure $3(\mathrm{a})$ and shows clearly the shape of the Ti $d$ orbitals. The main occupation comes from $d_{x z}$ and $d_{3 z 2-r 2}$. A small total magnetic moment is obtained for the unit cell, distributed among all the Ti atoms (see Figure 4).

3.2. $\mathrm{AlO}_{2} / \mathrm{TiO}-$ Hollow Interface. This interface (Figure 1(b)), presents a difference between LSDA and LSDA+U approximations. In LSDA $+\mathrm{U}$, the majority spin of the interfacial $\mathrm{Ti}$ $d_{x y}$ orbital shifts towards lower energies, so that the system becomes a semiconductor with a small gap of $0.1 \mathrm{eV}$ (see Figure 2(b)). This shift is smaller in the LSDA, so that the system is metallic. In Figure 3(b), we show the charge density at the anatase side of the interface where the $d_{x y}$ symmetry is evident. Both the charge and the magnetic moment at the Ti ions are large at the interface and decrease abruptly inside the anatase slab (see Figure 4).

3.3. $\mathrm{AlO}_{2} / \mathrm{TiO}$-Bridge Interface. In this interface (Figure $1(\mathrm{c})), E_{f}$ lies in the conduction band, and the La peaks are further away from it, overlapping the $t_{2 g}$ orbitals of the $\mathrm{Ti}$ atoms. The same result is obtained with LSDA. The interfacial Ti orbitals have different symmetry from those of the hollow case, being of $e_{g}$ character: $d_{x 2-y 2}$ and $d_{3 z 2-r 2}$, as can be seen in Figure 2 and Figure 3. In this case, the $e_{g}$ levels split into two regions: one occupied band close to $E_{f}$ and another one at energies above the $t_{2 g}$ orbitals, in the conduction band. The extra charge and the magnetic moment are both localized at the interface, near the oxygen vacancy, and their magnitude decreases abruptly inside the anatase slab as can be seen in Figure 4. In this case, the interfacial Ti formal valence and the magnetic moment are smaller than in the hollow structure.

It is interesting to note that in $\mathrm{AlO}_{2} / \mathrm{TiO}$ interfaces, there is no contribution of the $\mathrm{Ti}$ atom at the free surface in the occupied region of the conduction band, showing that a slab with 5 layers is a good enough approximation to account for interface properties.

There has been some controversy concerning the appearance of interfacial ferromagnetism in the LAO/STO interface 
[15], which may also be the case here. For this reason, we performed calculations with a double-size unit cell (54 atoms), for the $\mathrm{AlO}_{2} / \mathrm{TiO}$ interfaces which present localized magnetism at the interface. We studied the magnetic interaction between the interfacial Ti atoms within the LSDA+U method, setting them both in parallel and antiparallel configurations. The two solutions exist, but the antiparallel one is lower in energy, indicating that a long-range ferromagnetic interaction will not arise from the considered concentration and distribution of oxygen vacancies in $\mathrm{AlO}_{2} / \mathrm{TiO}$ interfaces. We do not exclude the possibility of ferromagnetism in $\mathrm{LaO} / \mathrm{TiO}_{2}$, but it would be minor, and due to the fact that all the $\mathrm{Ti}$ atoms have local magnetic moments, many magnetic configurations should be considered for this study.

To explore the consequences of a lower vacancy concentration, and also of a formally neutral system, we considered the double-size unit cell in the hollow configuration with only one interfacial oxygen vacancy. In this calculation, none of the $\mathrm{Ti}$ ions resulted $\mathrm{Ti}^{+3}$, and no magnetic moments appeared. Thus, a charge imbalance is needed to obtain $\mathrm{Ti}^{+3}$ ions and is also a necessary (but not sufficient) condition for the appearance of magnetism.

\section{Conclusions}

The principal conclusion of this work is that whatever the origin of the charge at the interfaces, its main effect is to change the valence of the $\mathrm{Ti}$ atoms, either if it is due to the layered structure of LAO or to the presence of oxygen vacancies in anatase.

In $\mathrm{AlO}_{2} / \mathrm{TiO}$, there are interfacial oxygen vacancies, and the interfacial $\mathrm{Ti}$ atom close to the vacancy position acquires an extra charge. In $\mathrm{LaO} / \mathrm{TiO}_{2}$, there are no oxygen vacancies as they are not electrostatically favored, and the extra charge is distributed among all $\mathrm{Ti}$ atoms, with decreasing value as the distance from the interface increases. Our results indicate that the $\mathrm{LaO} / \mathrm{TiO}_{2}$ interface spans through several anatase layers, while the $\mathrm{AlO}_{2} / \mathrm{TiO}$ one is more localized and presents large local magnetic moments.

In experimental samples, coexistence of the studied interfaces and possibly others as well is expected. As a consequence, small patches with different magnetic order might appear [16], thus giving rise to sample-dependent results.

\section{Acknowledgments}

The authors thank R. Weht for a careful reading of the paper. M. Weissmann and V. Ferrari are members of CONICET-Argentina and acknowledge support by Grant PIP-CONICET00038.

\section{References}

[1] P. Zubko, S. Gariglio, M. Gabay, P. Ghosez, and J. M. Triscone, "Interface physics in complex oxide heterostructures," Annual Review of Condensed Matter Physics, vol. 2, pp. 141-165, 2011.

[2] R. Pentcheva and W. E. Pickett, "Electronic phenomena atcomplex oxide interfaces: insights from first principles,"
Journal of Physics: Condensed Matter, vol. 22, no. 4, Article ID 043001, 2010.

[3] S. I. Kitazawa, Y. Choi, S. Yamamoto, and T. Yamaki, "Rutile and anatase mixed crystal $\mathrm{TiO}_{2}$ thin films prepared by pulsed laser deposition," Thin Solid Films, vol. 515, no. 4, pp. 19011904, 2006.

[4] S. A. Chambers, "Epitaxial growth and properties of doped transition metal and complex oxide films," Advanced Materials, vol. 22, no. 2, pp. 219-248, 2010.

[5] M. Weissmann and V. Ferrari, "Ab initio study of a $\mathrm{TiO}_{2} / \mathrm{LaAlO}_{3}$ heterostructure," Journal of Physics: Conference Series, vol. 167, no. 1, Article ID 012060, 2009.

[6] Z. Wang, W. Zeng, L. Gu, M. Saito, S. Tsukimoto, and Y. Ikuhara, "Atomic-scale structure and electronic property of the $\mathrm{LaAlO}_{3} / \mathrm{TiO}_{2}$ interface," Journal of Applied Physics, vol. 108, no. 11, Article ID 113701, 2010.

[7] P. Hohenberg and W. Kohn, "Inhomogeneous electron gas," Physical Review, vol. 136, no. 3B, pp. B864-B871, 1964.

[8] M. Weissmann, V. Ferrari, and A. Saul, "Ab initio study of magnetism at the $\mathrm{TiO}_{2} / \mathrm{LaAlO}_{3}$ interface," Journal of Materials Science, vol. 45, no. 18, pp. 4945-4951, 2010.

[9] P. Blaha, K. Schwarz, G. K. H. Madsen, D. Kvasnicka, and J. Luitz, WIEN2k, An Augmented Plane Wave + Local Orbitals Program for Calculating Crystal Properties, (Karlheinz Schwarz, Technology Universität Wien, Vienna, Austria, 2001.

[10] For the FP-LAPW calculations we use the following parameters: Atomic sphere radii of $1.7 \mathrm{Bohr}$ for Ti and $\mathrm{Al}, 1.4 \mathrm{Bohr}$ for $\mathrm{O}$ and 2.5 for La. The convergence control parameter RKmax is chosen to be 6 , that corresponds to an energy cutoff of $250 \mathrm{eV}$. The employed vacuum space between slabs is $20 \AA$.

[11] V. I. Anisimov, I. V. Solovyev, M. A. Korotin, M. T. Czyyk, and G. A. Sawatzky, "Density-functional theory and $\mathrm{NiO}$ photoemission spectra," Physical Review B, vol. 48, no. 23, pp. 16929-16934, 1993.

[12] M. Weissmann and R. Weht, "Electronic and magnetic properties of the different phases of $\mathrm{Ti}_{4} \mathrm{O}_{7}$ from density functional theory," Physical Review B, vol. 84, no. 14, Article ID 144419, 4 pages, 2011.

[13] In this work the number of vacancies considered is large, but the simulation of smaller vacancy concentrations would imply, due to the anatase structure, to start from corrugated interfaces that may be the the subject of future work.

[14] K. Janicka, J. P. Velev, and E. Y. Tsymbal, "Quantum nature of two-dimensional electron gas confinement at $\mathrm{LaAlO}_{3} / \mathrm{SrTiO}_{3}$ interfaces," Physical Review Letters, vol. 102, no. 10, Article ID 106803, 4 pages, 2009.

[15] M. R. Fitzsimmons, N. W. Hengartner, S. Singh et al., "Upper limit to magnetism in $\mathrm{LaAlO}_{3} / \mathrm{SrTiO}_{3}$ heterostructures," Physical Review Letters, vol. 107, no. 21, Article ID 217201, 5 pages, 2011.

[16] J. A. Bert, B. Kalisky, C. Bell et al., "Direct imaging of the coexistence of ferromagnetism and superconductivity at the $\mathrm{LaAlO}_{3} / \mathrm{SrTiO}_{3}$ interface," Nature Physics, vol. 7, pp. 767-771, 2011. 

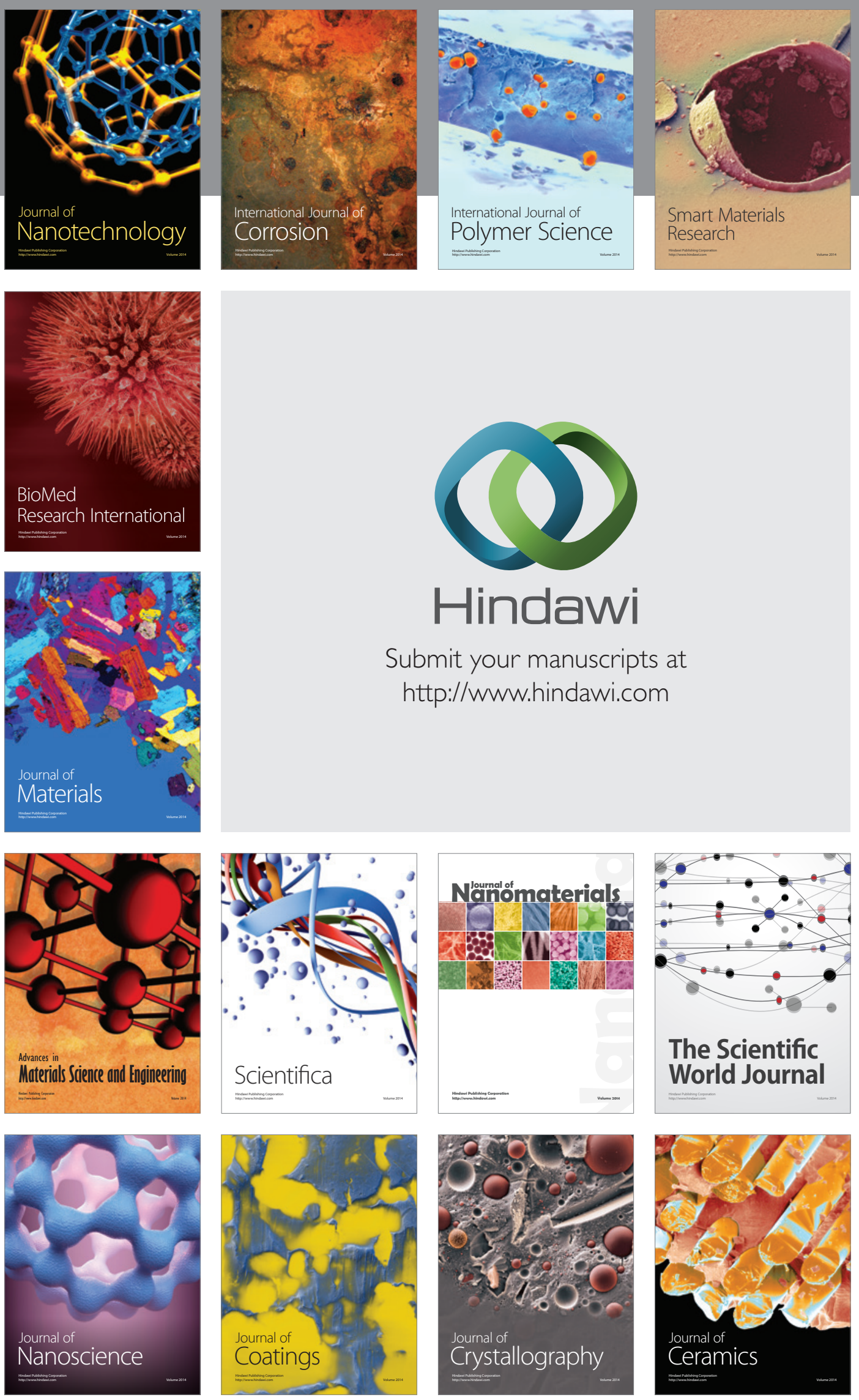

The Scientific World Journal

Submit your manuscripts at

http://www.hindawi.com

\section{World Journal}

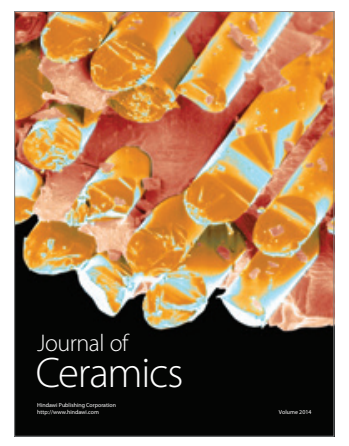

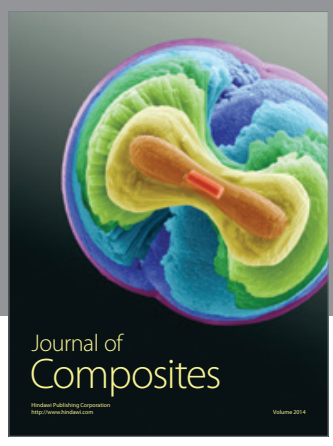
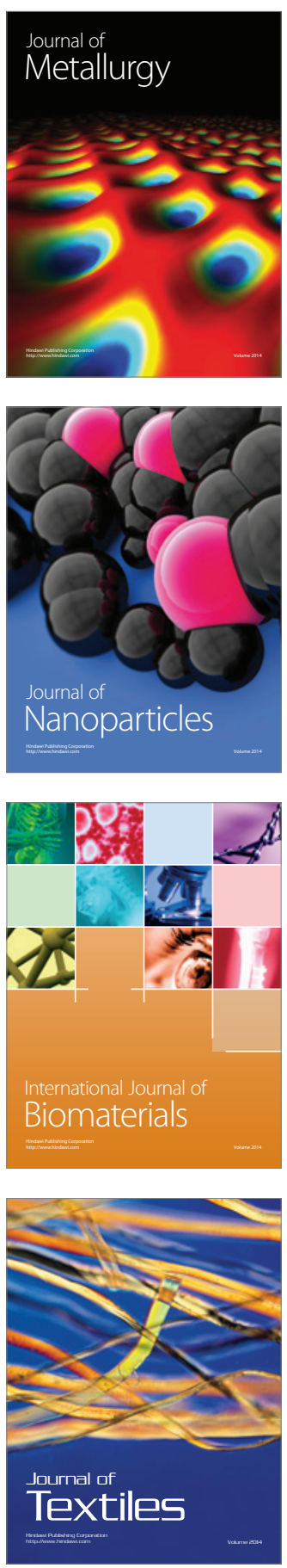